\title{
Interaction of Porosity with a Planar Solid/Liquid Interface
}

\author{
ADRIAN V. CATALINA, DORU M. STEFANESCU, SUBHAYU SEN, \\ and WILLIAM F. KAUKLER
}

In this article, an investigation of the interaction between gas porosity and a planar solid/liquid (SL) interface is reported. A two-dimensional numerical model able to accurately track sharp SL interfaces during solidification of pure metals and alloys is proposed. The finite-difference method and a rectangular undeformed grid are used for computation. The SL interface is described through the points of intersection with the grid lines. Its motion is determined by the thermal and solute gradients at each particular point. Changes of the interface temperature because of capillarity or solute redistribution as well as any perturbation of the thermal and solute field produced by the presence of non-metallic inclusions can be computed. To validate the model, the dynamics of the interaction between a gas pore and a solidification front in metal alloys was observed using a state of the art X-ray transmission microscope (XTM). The experiments included observation of the distortion of the SL interface near a pore, real-time measurements of the growth rate, and the change in shape of the porosity during interaction with the SL interface in pure $\mathrm{Al}$ and $\mathrm{Al}-0.25 \mathrm{wt}$ pet $\mathrm{Au}$ alloy. In addition, porosity-induced solute segregation patterns surrounding a pore were also quantified.

\section{INTRODUCTION}

GAS evolution during solidification is responsible for two casting defects: macroporosity (gas porosity) and microshrinkage (microporosity). While both defects have a significant influence on mechanical properties, their mechanism of formation is quite different. ${ }^{[1]}$ Macroporosity results when gas is rejected from the liquid and is entrapped in the solidifying metals as spherical gas bubbles of millimeter size. Microshrinkage occurs when liquid metal cannot reach interdendritic areas during casting solidification, and is caused by a combination of shrinkage and gas evolution. It is a standard foundry defect for mushy-freezing alloys, such as aluminum alloys, steel, superalloys, brass, bronze, and cast iron. Its size is of the order of 10 to $100 \mu \mathrm{m}$.

Previous experimental studies of porosity evolution in metals have primarily relied on quenching of directionally solidified samples followed by postsolidification analysis of the combined effect of gas and shrinkage porosity. For example, experiments performed on $\mathrm{Cr}, \mathrm{Ni}$, and Mo alloyed stee ${ }^{[2]}$ revealed that elongated or ellipsoidal macroporosity occurred only when a nucleation agent such as $\mathrm{Al}_{2} \mathrm{O}_{3}$ was added to the melt. In the absence of nucleants, irrespective of the hydrogen content, only microshrinkage was obtained. The effect of gravity on pore distribution was studied by Kim et $a l^{[3]}$ in a $\mathrm{A} 356 \mathrm{Al}-\mathrm{Si}$ alloy. They demonstrated that for upward solidification, the distribution of pores was concentrated at the hot (upper) end of the sample, implying that terminal floatation velocity was the dominant factor. Floatation did not permit interaction between the pores and the solid/liquid (SL) interface. During downward solidification,

ADRIAN V. CATALINA, Scientist, and SUBHAYU SEN, Senior Scientist, are with BAE SYSTEMS Analytical Solutions, NASA Marshall Space Flight Center. Huntsville, AL 35811. Contact e-mail: adrian.catalina@msfc.nasa.gov DORU M. STEFANESCU, Cudworth Professor of Metallurgical and Materials Engineering, is with The University of Alabama, Tuscaloosa, AL 35487 and WILLIAM F. KAUKLER, Associate Research Professor, is with The University of Alabama in Huntsville, Huntsville, AL 35899.

Manuscript submitted June 5, 2002. both macro- and microshrinkage were found entrapped in the mushy zone. However, postsolidification analysis is incapable of either capturing the dynamics of porosity evolution or their interaction with the solidification front.

To overcome this lack of real-time data, Lee and Hunt ${ }^{[4]}$ used a microfocus X-ray system with a resolution of $25 \mu \mathrm{m}$ to study the real-time interaction between a pore and primary dendrites in $\mathrm{Al}-10$ wt pct $\mathrm{Cu}$ samples. Their experimental observation revealed that the shape of the pore undergoes a transition from spherical to ellipsoidal once the pore is impinged upon by the dendritic interface. With an increase in growth velocity, the primary dendrite arm spacing decreases and further elongates the pore in the growth direction. However, this investigation did not address the issue of solutal segregation that may be induced by the porosity during solidification.

Real-time observations on the interaction between the solidification front and a pore have also been performed on transparent organic (analogue) materials. Jamgotchian et al. $^{[5]}$ demonstrated that in the succinonitrile-acetone system, gas bubbles that formed at the planar SL interface grew in a coupled fashion into elongated shapes called duplexes. Experiments with the faceted salol system have shown that there exists a critical solidification velocity at which a bubble of given radius is engulfed. ${ }^{[6]}$ Although these experiments have provided real-time observations of solidification dynamics, they do not necessarily reflect the behavior in a metallic system. Unlike salol, most metallic systems are nonfaceted. In addition, metallic systems have much higher interfacial energy compared to organic analogues. Further, the chemistry between a metal-gas system such as $\mathrm{Al} / \mathrm{H}_{2}$ is very different from an organic-organic system such as succinonitrile and acetone, where acetone is the source of pore formation. It must also be emphasized that experiments performed with organic analogues are typically carried out in thin slabs with characteristic thickness in the range of 10 to $300 \mu \mathrm{m}$. Consequently, the effect of wall friction on pore nucleation and distribution can be significant, resulting in controversial interpretations.

The experimental objective of this study is to isolate the interaction between a gas pore and a planar SL interface in metallic systems. The understanding obtained from such an 
investigation can be directly transferred to the distribution of interdendritic gas porosity and solutal segregation associated with it in castings solidification. To this goal, horizontal directional solidification (HDS) experiments with pure $\mathrm{Al}$ and $\mathrm{Al}-$ 0.25 wt pct Au were conducted under direct and real-time $\mathrm{x}$-ray transmission microscope (XTM) observation. This technique permits real-time tracking of the change in shape and size of porosity and the localized porosity-induced solutal segregation.

Numerous models have been proposed over the years to model microporosity in castings. Piwonka and Fleming ${ }^{[7]}$ have elegantly modeled the interplay between gas and shrinkage porosity. They essentially showed that the effect of dissolved gas and shrinkage on pore formation is additive. Higher surface tension of the melt, on the other hand, can significantly increase the critical amount of dissolved gas required for gas porosity formation. Poirier et al. ${ }^{[8]}$ improved the model by calculating the local pressure in the mushy zone as a function of columnar dendritic microstructural features and the corresponding permeability and fluid flow in the interdendritic region. Lee and Hunt ${ }^{[4,9]}$ used a continuum model to describe their experimental results in the $\mathrm{Al}-\mathrm{Cu}$ alloy. They allowed pore growth by consumption of hydrogen from a supersaturated control volume. At each timestep, the pore radius is compared to dendritic spacing to determine the shape of the pore. Voller and Sundarraj ${ }^{[10]}$ and later Rousset et al. ${ }^{[1]}$ modeled the effect of microporosity on solutal segregation by coupling an inverse segregation model with a microporosity model. They demonstrated that since inverse segregation is directly related to interdendritic fluid flow, the presence of microporosity decreases the extent of inverse segregation in the mushy zone. However, none of these models describes the exact physics that correlate solidification velocity with the SL interface shape as it interacts with a pore and the localized solutal segregation induced by the pore. In this article, we will address these issues from a modeling standpoint.

The theoretical problem is to accurately track the sharp and arbitrarily shaped SL interfaces. Finding an adequate solution to this problem is an important and necessary step, because it can open new possibilities to study the microscale phenomena occurring during the solidification. Morphological stability, coarsening processes, and SL interface interaction with gaseous or non-metallic inclusions are only a few examples of phenomena that existing numerical solidification models can describe with various degrees of accuracy. Among the various techniques developed to date, the boundary-fitted coordinates, ${ }^{[12,13,14]}$ phase field, ${ }^{[15,16,17]}$ and level set ${ }^{[18,19]}$ methods are the most notable. However, the boundary-fitted coordinates method experiences difficulties when dealing with highly distorted interfaces. It requires remeshing as the interface changes its shape. The versatility of the phase-field method lies in the fact that it can use a simple finite-difference technique, use a fixed grid, and does not require remeshing. However, despite tremendous efforts to improve the computational efficiency, ${ }^{[20,21]}$ the method is still computationally intensive and produces only diffuse interfaces. The level set method was first introduced by Osher and Sethian ${ }^{[18]}$ and, since then, has been used to solve numerous problems ${ }^{[22-27]}$ including solidification. The method is built on the idea that a moving interface separating two phases can be represented as the zero contour of a function $\omega$, which is the level set function. In general, the function $\omega$ is defined as the signed normal distance from the interface to each of the grid points on the computational lattice. It has its own equation of motion represented by

$$
\frac{\partial \omega}{\partial t}+F|\nabla \omega|=0
$$

where $t$ is time and $F$ is the normal velocity field, which is variable in space and time. A number of ways were proposed to construct the normal velocity field, $F$. A boundary integral approach was initially used by Sethian and Strain. ${ }^{[19]}$ Then, faster algorithms based on partial differential equations were developed. ${ }^{[26]}$ More recently, Kim et al. ${ }^{[27]}$ applied an even simpler method by calculating the velocity field from the difference in the normal derivatives of the temperature field at the interface. In essence, the level set method is conceptually similar to the phase field method in that neither one explicitly tracks the interface.

Udaykumar and co-workers ${ }^{[28,29,30]}$ have proposed an interface tracking technique based on a finite volume formulation. The moving SL interface is represented by a discrete set of markers that are positioned at the intersection of the interface with the grid lines. In this manner, after each iteration, only the interface must be reconstructed. However, the requirement of redefinition of the control-volumes near the interface considerably increases the difficulty of model implementation and makes it susceptible to numerical errors.

In the present article, we propose a numerical technique based on the Udaykumar and Shyy model. It uses the finitedifference formulation, thus eliminating the need for controlvolume redefinition at the interface. ${ }^{[31]}$ The proposed approach requires calculations of the normal velocity only at the SL interface. This is in contrast to the level set method where extension and reinitialization of the normal velocity off the interface is necessary.

\section{MODEL FORMULATION}

\section{A. Interface Tracking}

Consider a SL interface, as shown in Figure 1. At the beginning of a time-step, the interface is described by a discrete series of markers positioned at the intersection of the SL interface with the grid lines. The open circles in Figure 1 represent these markers. During the time-step, the SL interface advances according to the relative values of the

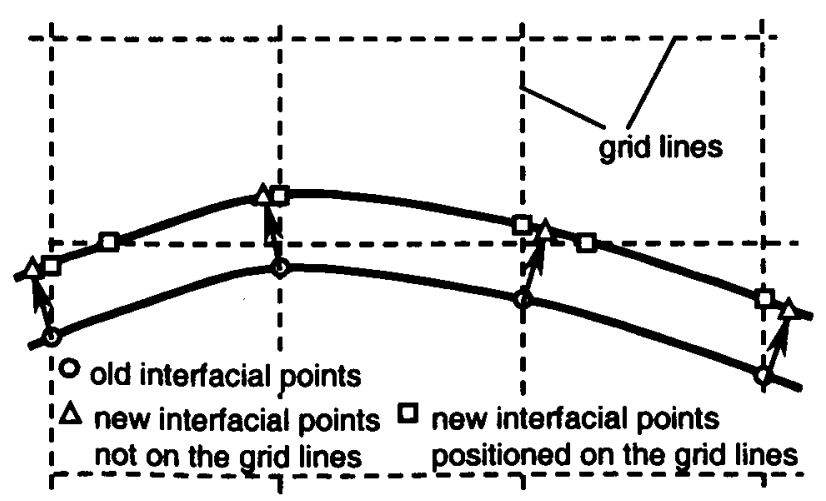

Fig. 1-Definition of the interfacial points. 
temperature and solute gradients in the liquid and solid phases. These gradients and the corresponding local solidification velocity are calculated at the marker points by following a procedure that will be explained latter. At the end of this new time-step, the SL interface markers will find themselves in positions other than the intersection with the grid lines. These are represented by the open triangles in Figure 1. Consequently, a new set of markers, on the grid lines (the open squares), must be generated based on the positions of the old marker points. This task is done by a simple intersection procedure. This procedure requires the marker points to be numbered in increasing order, such that the liquid lies always to the left and the solid to the right as one advances along the interface in the direction of the arrow, as shown in Figure 2. Then, to calculate the intersection of the SL interface with the grid lines passing between the points $i-1$ and $i$, a variety of methods can be employed. Udaykumar and Shyy ${ }^{[30]}$ have used segments of circular arcs to describe the SL interface between three consecutive markers. In a previous work by two of the authors of this paper, ${ }^{[32]}$ second-degree polynomials were fitted through three consecutive markers, while in the present formulation, the cubic spline interpolation procedure ${ }^{[33]}$ is used to find the position of the new marker points.

\section{B. Local Interfacial Gradients/Fluxes and Local Solidification Velocity}

The local solidification velocity, i.e., the local rate at which the SL interface moves, is determined by the interfacial fluxes of the transport variables (heat and solute) calculated at the marker points. Since these fluxes are normal to the SL interface, one needs to calculate first the normal direction at each marker point. The direction of the unit normal vector, $\mathbf{N}$, to a curve $y=y(x)$, is given by

$$
\mathbf{N}_{i}=\frac{1}{\sqrt{1+(d y / d x)^{2}}}\left[-\left(\frac{d y}{d x}\right)_{i} \cdot \mathbf{i}+1 \cdot \mathbf{j}\right]
$$

where the subscript $i$ denotes that the calculation is performed at the interfacial point $i$, and $\mathbf{i}$ and $\mathbf{j}$ are the unit vectors along the $x$ and $y$ directions, respectively. Also, the function $y(x)$ can be constructed as a cubic polynomial fitted through four consecutive interfacial marker points. Because the interfacial marker points are numbered according to the rule established in the previous section, the normal calculated through Eq. [2] will point always into the liquid phase. By simply changing the sign in Eq. [2], the normal will point into the solid phase.

Consider now an interface segment containing the marker $i$. The gradient of a field variable $\Phi$ (temperature, solute con-

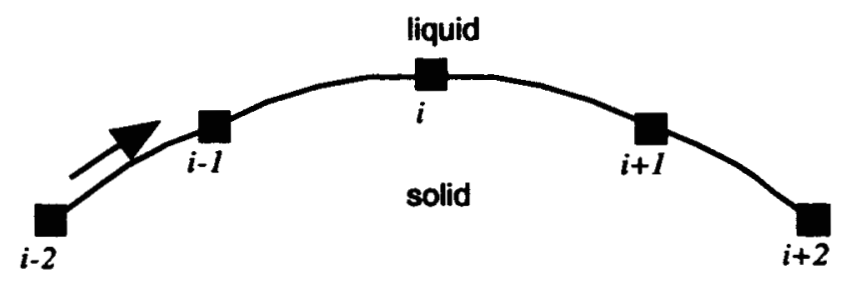

Fig. 2-Marker points numbering along the SL interface. centration) at point $i$ is calculated based on the following equation (also Figure 3):

$$
\frac{\partial \Phi}{\partial n} \cong \frac{\Phi_{i+\Delta n}-\Phi_{i}}{\Delta n}
$$

where $\Delta n$ is the length of a probe projected from the interfacial point $i$ along the direction of the normal vector $\mathbf{N}$ into the adjacent liquid and solid phases, and $\Phi_{i}$ and $\Phi_{i+\Delta n}$ are the values of the field variables at the locations $i$ and $i+\Delta n$, respectively. The problem when using Eq. [3] is that the location $i+\Delta n$ usually does not fall onto a grid point, and therefore, the value $\Phi_{i+\Delta n}$ must be obtained by using some kind of interpolation technique. The interpolation function for plane bilinear isoparametric elements, very popular in the finite-element method (for example, Reference 34), has been used in this work. This type of interpolation function is of the form $\Phi(x, y)=a+b \cdot x+c \cdot y+d \cdot x \cdot y$, where the coefficients $a, b, c$, and $d$ can be obtained by choosing four grid points in the same phase around the location $i+\Delta n$ (Figure 4). Then, the field variable at the location $i+\Delta n$ characterized by the local coordinates $\left(\xi_{n}, \eta_{n}\right)$ is given by

$$
\Phi_{i+\Delta n}=\sum_{p=1}^{4} N_{p} \Phi_{p}
$$

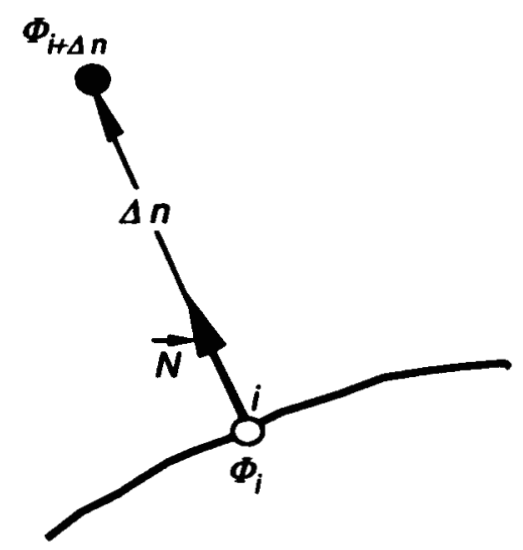

Fig. 3-Calculation of the interface gradient of a field variable.

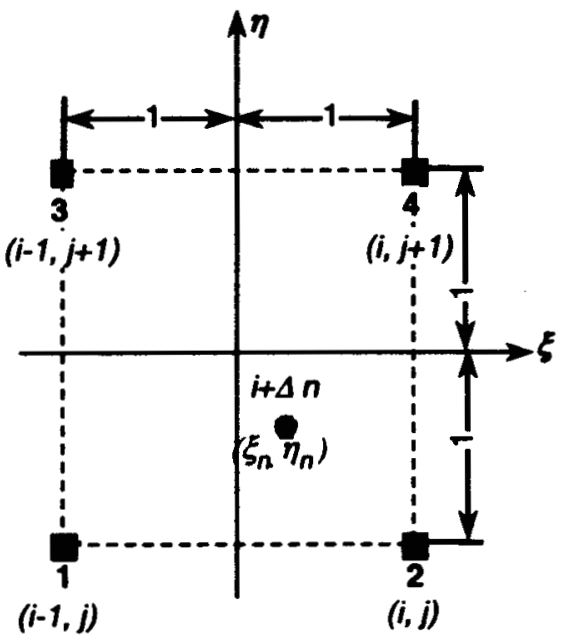

Fig. 4 Development of the interpolation function. 
where $\Phi_{p}$ is the value of the field variable at the node $p$ ( $p=1-4$, Figure 4 ), and $N_{p}$, represents the shape functions defined as ${ }^{[32,34]}$

$$
\left\{\begin{array}{l}
N_{1}=\frac{1}{4}\left(1-\xi_{n}\right)\left(1-\eta_{n}\right) \\
N_{2}=\frac{1}{4}\left(1+\xi_{n}\right)\left(1-\eta_{n}\right) \\
N_{3}=\frac{1}{4}\left(1-\xi_{n}\right)\left(1+\eta_{n}\right) \\
N_{4}=\frac{1}{4}\left(1+\xi_{n}\right)\left(1+\eta_{n}\right)
\end{array}\right.
$$

A necessary requirement for interpolation is that the four grid points surrounding the location $i+\Delta n$ must pertain to the same phase. In order to ensure this requirement, the length, $\Delta n$, of the projected normal is chosen as

$$
\Delta n=\sqrt{\Delta x^{2}+\Delta y^{2}}
$$

where $\Delta x$ and $\Delta y$ are the grid size along the $x$ and $y$ directions, respectively.

The interfacial temperature, $T_{i}$, at a location $i$ is given by the relationship

$$
T_{i}=T_{M}+m_{L} \cdot\left(C_{L}\right)_{i}+\Gamma \cdot K_{i}
$$

where $T_{M}$ is the melting temperature of the pure base metal, $m_{L}$ is the liquidus slope, $\left(C_{L}\right)_{i}$ is the solute concentration at the interface in the liquid phase, $\Gamma$ is the Gibbs-Thomson coefficient, and $K_{i}$ is the SL interface curvature at the location $i$. The term $K_{i}$ can be calculated as

$$
K_{i}=\frac{\left(d^{2} y / d x^{2}\right)_{i}}{\left[1+(d y / d x)_{i}^{2}\right]^{3 / 2}}
$$

where, again, $y=y(x)$ is the local shape of the SL interface. The interfacial solute concentration in the solid phase, $\left(C_{S}\right)_{i}$, is related to the liquid concentration, $\left(C_{L}\right)_{i}$, through the relationship

$$
\left(C_{L}\right)_{i}=\frac{\left(C_{S}\right)_{i}}{k}
$$

where $k$ is the solute partition coefficient between the solid and the liquid phase.

The local solidification velocity, $\left(V_{n}\right)_{i}$, normal to the interface at each marker point, $i$, can be calculated from heat and mass balance at the interface, as follows:

$$
\begin{gathered}
\left(V_{n}\right)_{i} C_{p}(d T)_{i}=k_{L}\left(\frac{\partial T_{L}}{\partial n}\right)_{i}+\left(V_{n}\right)_{i} \Delta H_{f}+k_{S}\left(\frac{\partial T_{S}}{\partial n}\right)_{i} \\
-D_{L} \cdot\left(\frac{\partial C_{L}}{\partial n}\right)_{i}=\left(V_{n}\right)_{i}(1-k) \cdot\left(C_{L}\right)_{i}
\end{gathered}
$$

where $C_{p}$ is the volumetric specific heat of the solidifying alloy; $k_{L}$ and $k_{S}$ are the thermal conductivities in the liquid and solid phase, respectively; $\Delta H_{f}$ is the volumetric heat of fusion; $D_{L}$ is the solute diffusion coefficient in the liquid; and $\left(\partial \Phi_{j} / \partial n\right)_{i}$ is the interface gradient of the field variable (temperature, $T$, and concentration, $C$ ) in the phase $j$ (solid, $S$, and liquid, $L$ ). Note that the sign convention used for the gradient terms in Eq. [10] is defined by Eq. [3]. Also, in Eq. [10], the quantity $(d T)_{i}$ represents the change of the interface temperature at the location $i$ in a time $d t$ as a result of the interface curvature and concentration change during the time $d t$. The effect of solute diffusion in the solid phase has been ignored in Eq. [11].

\section{Calculation of Field Variables}

The field variables can be calculated by solving the transport equations separately in the liquid and solid phases. Thus,

$$
\frac{\partial \Phi}{\partial t}=\Xi \cdot \nabla^{2} \Phi
$$

where $\Xi$ is the generalized diffusivity, i.e., thermal diffusivity in the solid, $\alpha_{S}$, and in the liquid, $\alpha_{L}$, as well as mass diffusivity, $D_{S}$ and $D_{L}$, in the solid and liquid, respectively. Equation [12] can be solved by using a finite difference method.

When discretizing Eq. [12], a nonuniform grid size must be considered. Even if a uniform grid is used to discretize the computational domain, a nonuniform spacing appears in the proximity of the SL interface. This situation is illustrated in Figure 5. By using an implicit formulation, the finite differences expression of Eq. [12] can be written as

$$
\begin{aligned}
\frac{\Phi_{i, j}^{n}-\Phi_{i, j}^{o}}{\Delta t}= & \Xi \cdot\left[b_{W} \Phi_{i-1, j}^{n}+b_{N} \Phi_{i, j+1}^{n}+b_{E} \Phi_{i+1, j}^{n}\right. \\
& \left.+b_{S} \Phi_{i, j-1}^{n}-\left(b_{x}+b_{y}\right) \Phi_{i, j}^{n}\right]
\end{aligned}
$$

where the superscripts $n$ and $o$ indicate the value of the field variable at the end and at the beginning of the time-step, respectively, and $\Delta t$ is the size of the time-step. With the

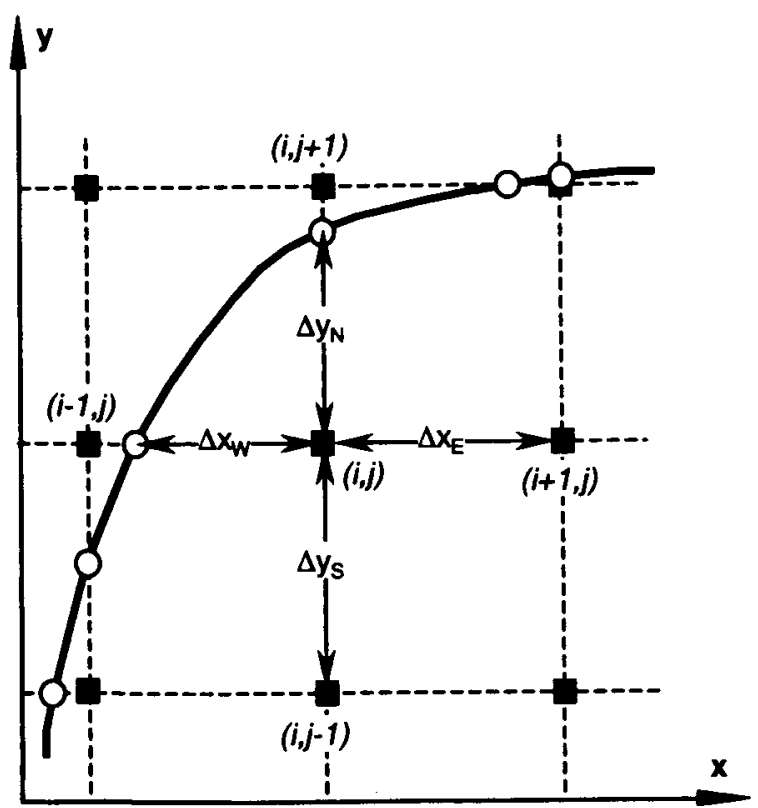

- grid points, $\mathrm{O}$ - interface markers

Fig. 5-Nonuniform spacing in the vicinity of the SL interface. 
notations used in Figure 5, the coefficients $b_{W}, b_{N}, b_{E}, b_{S}$, $b_{x}$, and $b_{y}$ in Eq. [13] are as follows:

$$
\begin{aligned}
b_{W} & =\frac{2 \Delta x_{E} / \Delta x_{W}}{\Delta x_{E} \Delta x_{W}\left(1+\Delta x_{E} / \Delta x_{W}\right)} ; \\
b_{E} & =\frac{2}{\Delta x_{E} \Delta x_{W}\left(1+\Delta x_{E} / \Delta x_{W}\right)} \\
b_{S} & =\frac{2 \Delta y_{N} / \Delta y_{S}}{\Delta y_{N} \Delta y_{S}\left(1+\Delta y_{N} / \Delta y_{S}\right)} ; \\
b_{N} & =\frac{2}{\Delta y_{N} \Delta y_{S}\left(1+\Delta y_{N} / \Delta y_{S}\right)} \\
b_{x} & =\frac{2}{\Delta x_{E} \Delta x_{W}} ; \quad b_{y}=\frac{2}{\Delta y_{N} \Delta y_{S}}
\end{aligned}
$$

To solve Eq. [13], boundary conditions on the SL interface and sample walls are required. Here, we must point out that Eq. [7] through Eq. [9] do not unequivocally define the boundary conditions on the interface, unless the solidification of a pure substance is considered. The reason is that these equations do not account for the change of the interface concentration, $\left(C_{L}\right)_{i}$, during the solidification process. Except for simple cases, such as directional solidification with a planar SL interface ${ }^{[35]}$ there are no analytical solutions to describe this change. However, to account for the evolution of $\left(C_{L}\right)_{i}$, the following methodology has been used in the present work. It relies on the assumption that the local quantities $\Phi_{i+\Delta n}$ (temperature, concentration) as well as the local curvature, $K_{i}$, do not change significantly as a segment of the SL interface moves over the distance $\left(V_{n}\right)_{i} \cdot \Delta t$. Therefore, the local change of the SL interface temperature, $\Delta T_{i}$, during the time $\Delta t$ is given by

$$
\Delta T_{i}=m_{L}\left\lfloor\left(C_{L}\right)_{i}-\left(C_{L}\right)_{i}^{o}\right\rfloor
$$

where $\left(C_{L}\right)_{i}^{\mathrm{o}}$ is the concentration at the beginning of the time-step. In addition, the finite difference expressions for the balance equations (Eq. [10] and Eq. [11]) can be written as

$$
\begin{gathered}
\left(V_{n}\right)_{i} C_{p} \Delta T_{i}=\frac{k_{L}\left\lfloor T_{n L}-\left(T_{i}^{\mathrm{o}}+f \Delta T_{i}\right)\right\rfloor}{\Delta n}+\left(V_{n}\right)_{i} \Delta H_{f} \\
-\frac{k_{S}\left\lfloor\left(T_{i}^{\mathrm{o}}+f \Delta T_{i}\right)-T_{n S}\right\rfloor}{\Delta n} \\
\left(V_{n}\right)_{i}=D_{L} \frac{\left(C_{L}\right)_{i}-C_{n L}}{\Delta n(1-k)\left(C_{L}\right)_{i}}
\end{gathered}
$$

where $T_{i}^{\mathrm{o}}$ is the local interface temperature at the beginning of the time-step; $T_{n L}$ and $C_{n L}$ are the temperature and concentration, respectively, at the location $i+\Delta n$ into the liquid phase; $T_{n}$ is the temperature similarly defined in the solid phase; and $f$ is a factor that takes the value of 0.5 to account for average temperature gradients at the interface during the time-step. Further, by eliminating $\left(C_{L}\right)_{i}$ from Eq. [17] and replacing it in Eq. [16] by making use of Eq. [15], the following second degree equation can be obtain for the local growth velocity $\left(V_{n}\right)_{i}$ :

$$
A_{1}\left(V_{n}\right)_{i}^{2}-A_{2}\left(V_{n}\right)_{i}+A_{3}=0
$$

with

$$
\left\{\begin{array}{l}
A_{1}=\left\lfloor\Delta H_{f}+C_{P} m_{L}\left(C_{L}\right)_{i}^{\mathrm{o}}\right\rfloor(1-k) \Delta n \\
A_{2}=\left[\Delta H_{f}+C_{P} m_{L}\left(\left(C_{L}\right)_{i}^{\mathrm{o}}-C_{n L}\right)\right] D_{L}-Q(1-k) \Delta n \\
A_{3}=\left[\frac{f m_{L}\left(k_{S}+k_{L}\right) C_{n L}}{\Delta n}-Q\right] D_{L} \\
Q=\frac{f m_{L}\left(k_{S}+k_{L}\right)\left(C_{L}\right)_{i}^{\mathrm{o}}}{\Delta n}+k_{L} \frac{T_{n L}-T_{i}^{\mathrm{o}}}{\Delta n}-k_{S} \frac{T_{i}^{\mathrm{o}}-T_{n S}}{\Delta n}
\end{array}\right.
$$

The only valid solution of Eq. [18] is

$$
\left(V_{n}\right)_{i}=\frac{A_{2}-\sqrt{A_{2}^{2}-4 A_{1} A_{3}}}{2 A_{1}}
$$

Then, $\left(C_{L}\right)_{i}$ and $T_{i}$ can be calculated from Eqs. [17] and [7], respectively. The boundary conditions on the SL interface and the solidification velocity are thus calculated. It must be added here that although the probability is very small, in some cases, it is still possible for an interface marker point to fall on a grid point, thus making at least one coefficient in Eq. [14] take an infinite value. For such special situations, the particular grid point located on the SL interface is treated as a boundary point. While the temperature of this bouindary point is the same on either side, the concentration is different on the side facing the liquid phase from that on the side facing the solid phase (Eq. [9]).

\section{Nondimensional Equations and Time-Step}

In order to minimize the round-off errors, a nondimensional form of the constitutive equations presented in this section must be used. Because for all the case studies considered in this work an initial solidification velocity, $V$, was imposed through the external boundary conditions, this velocity was taken as a reference velocity, $V_{\text {ref }}$. The reference time scale, $t_{\text {ref }}$, then follows from the relationship $t_{\text {ref }}=L_{\text {ref }} / V_{\text {ref }}$, where $L_{\text {ref }}$ is the characteristic lengthscale of a particular case. The other reference quantities used in this work are the melting/solidus temperature of the solidifying material, liquid thermal and solutal diffusivities, liquid thermal conductivity, and the initial concentration of the melt.

Because the SL interface is explicitly tracked in this model, restrictions must be imposed on the size of the timestep in order to ensure stability. A variable time-step of size given by the following empirical relationship has been used in this work:

$$
\Delta t=\frac{1}{2 \cdot V_{\max }} \sqrt{\frac{\Delta y^{3} \cdot V}{\alpha_{L}}}
$$

where $V_{\max }$ is the instantaneous maximum solidification velocity in the system and $V$ is the imposed solidification velocity.

\section{MODEL INTERROGATION}

In Sections A and B, a number of case studies are considered in order to test the capabilities and the accuracy of the proposed numerical model. Whenever possible, analytical solutions will also be presented for comparison. The thermophysical properties used in the calculations are presented in Table I. 
Table I. Thermophysical Data Used in Calculations

\begin{tabular}{|c|c|c|c|c|c|c|c|}
\hline \multicolumn{2}{|l|}{ Property } & Al & $\mathrm{Al}-2 \mathrm{Wt}$ Pct $\mathrm{Cu}$ & Al-0.25 Wt Pct $\mathrm{Au}$ & Sn-0.5 At. Pct Bi & $\mathrm{ZrO}_{2}$ & $\mathbf{H}_{2}$ \\
\hline \multicolumn{2}{|l|}{ Heat of fusion ${ }^{[41,42]}\left(\mathrm{J} / \mathrm{m}^{3}\right) \cdot 10^{8}$} & 9.5 & $9.5^{*}$ & $9.5^{*}$ & $4.179^{*}$ & - & - \\
\hline \multicolumn{8}{|l|}{ Gibbs-Thomson coefficient ${ }^{[41]}$} \\
\hline$(\mathrm{K} \cdot \mathrm{m}) \cdot 10^{-7}$ & & 0.9 & $0.9^{*}$ & $0.9 *$ & - & - & - \\
\hline Liquidus slope $^{[41,43,44]}(\mathrm{K} / \mathrm{wt} \%)$ & & - & -2.6 & -1.43 & $-1.95^{* *}$ & - & - \\
\hline Partition coefficient ${ }^{[41,43]}$ & & - & 0.14 & 0.048 & 0.28 & - & - \\
\hline \multicolumn{7}{|l|}{ Solute diffusivity in the } & - \\
\hline Thermal conductivity ${ }^{[41,42,46,47]}$ & liquid & 95 & $95^{*}$ & $95^{*}$ & 30 & 1.97 & 0.43 \\
\hline$(\mathrm{W} / \mathrm{m} \cdot \mathrm{K})$ & solid & 210 & $210^{*}$ & $210^{*}$ & 60 & & \\
\hline Specific heat ${ }^{[41,42,47,48]}$ & liquid & 2.58 & $2.58 *$ & $2.58 *$ & $1.81^{*}$ & $3.63 \cdot 10^{6}$ & 387.92 \\
\hline$\left(\mathrm{J} / \mathrm{m}^{3} \cdot \mathrm{K}\right) \cdot 10^{6}$ & solid & 3 & $3 *$ & $3^{*}$ & $1.96^{*}$ & & \\
\hline
\end{tabular}

*Property assumed to be the same as that of the base metal.

$* *$ K/at. pet.

\section{A. Solidification of Pure Aluminum in the Vicinity of a Particle}

Directional solidification of pure aluminum in the vicinity of a spherical $\mathrm{ZrO}_{2}$ particle of radius $R_{p}=22.5 \mu \mathrm{m}$ was chosen as a first case study. The imposed solidification velocity was $V=2 \mu \mathrm{m} / \mathrm{s}$ under a thermal gradient in the liquid $G_{L}=7 \mathrm{~K} / \mathrm{mm}$. The calculations were performed on a sample of length $L=2 \mathrm{~mm}$ and a width $W$, which was varied, from case to case, from 1 to $0.4 \mathrm{~mm}$ in order to test its influence on the evolution of the SL interface shape. However, no noticeable effect was observed. At the beginning of the calculations (i.e., $t=0$ ), it was considered that at one end of the sample a slice of $\Delta z=10 \mu \mathrm{m}$ thickness was in solid state and that the SL interface was perfectly planar. The center of the particle was placed at $x=W / 2$ and $z=\Delta z+h_{c}$, where $h_{c}$ is the distance between the particle center and the planar SL interface (Figure 6). Insulated boundary conditions were used on the $x$ direction, while at the two ends of the sample, constant cooling rates were considered (also Figure 6). The cooling conditions were set such as to maintain the imposed solidification velocity, $V$, of the originally planar interface for the entire duration of the calculations. They were $\partial T / \partial t=-V \cdot G_{L}$ at the liquid end and $\partial T / \partial t=-V \cdot G_{S}$ at the solid end, where $G_{S}$ is the temperature gradient in the solid phase.

The available analytical solution for a problem of this type ${ }^{[36]}$ is presented in Appendix I together with some scaling considerations. This solution predicts that when the thermal conductivity of the particle, $k_{P}$, is smaller than that of the liquid, $k_{L}$, the SL interface will become convex toward the particle, forming a bump as it approaches the particle. Indeed, our numerical calculations show this behavior, as can clearly be observed in Figures 7 and 8.

Figure 7 shows a comparison between the numerical and the analytical solutions when neglecting the Gibbs-Thomson effect (i.e., $\Gamma=0$ ). The agreement is good, thus proving the capability of the proposed interface tracking model. In all our numerical simulations, the interface perturbation is somewhat sharper than that predicted by the analytical solution. This is mainly because of the insulated boundary conditions used at a finite distance from the particle (Figure 6) as compared to the analytical solution, which implies that $\partial T / \partial x=0$ occurs at $x \rightarrow \infty$.

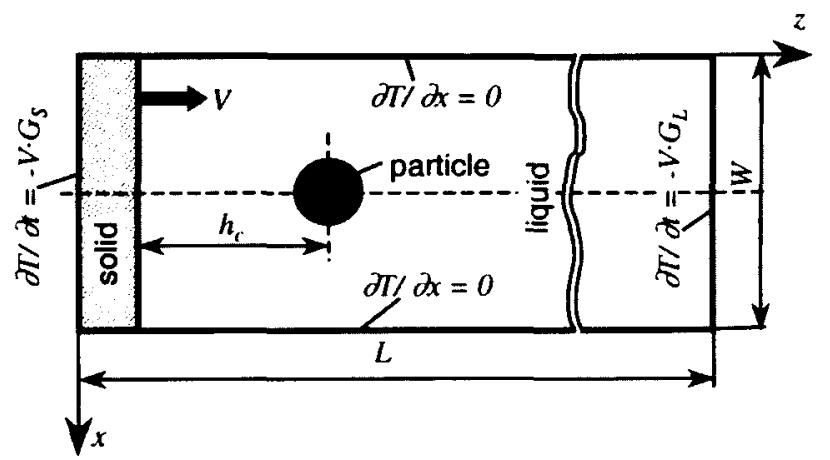

Fig. 6-The geometry and boundary conditions for the sample used in computations.

Figure 8 shows the shape of the SL interface when accounting for the Gibbs-Thomson effect. It can be observed that, as expected, the amplitude of the interface perturbation becomes considerably smaller as compared to the case when $\Gamma=0$. The influence of the temperature gradient, $G_{L}$, is also shown in this figure. In qualitative agreement with Eq. [Ic] (Appendix I), the numerical calculations show that, for the same distance $h_{c}$, as $G_{L}$ increases, the perturbation becomes sharper and its amplitude also increases. This implies that the perturbation develops faster when increasing $G_{L}$.

It also has to be mentioned here that when accounting for the Gibbs-Thomson effect, the outcome of the calculations can be influenced to some extent by the choice of the grid size on which the calculated curvature is strongly dependent. A procedure to estimate the level of errors introduced by a certain grid size is presented in Appendix II. Although the developed model allows the use of a nonuniform grid, a uniform grid of size $R_{P} / 6$ on both $x$ and $y$ directions was used to produce the preceding results. For all the other problems that will be discussed in this article, This paper unless specified otherwise, the same grid size has been employed.

\section{B. Solidification of Alloys; the Influence of Solute}

The first test problem that we consider in this case is the solute redistribution during the initial transient of plane front directional solidification of a binary alloy. An analytical 


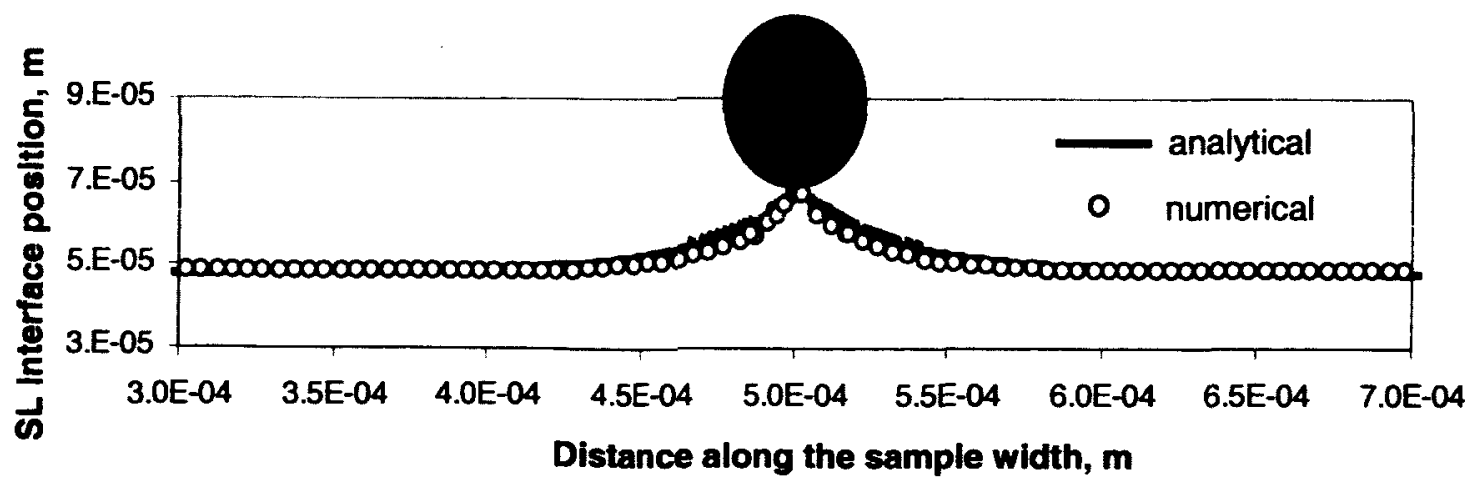

Fig. 7-SL interface shape: comparison of analytical and numerical solutions (pure $\mathrm{Al}, \mathrm{ZrO}_{2}$ particle, $R_{p}=22.5 \mu \mathrm{m}, V=2 \mu \mathrm{m} / \mathrm{s}$, with no Gibbs-Thomson effect).

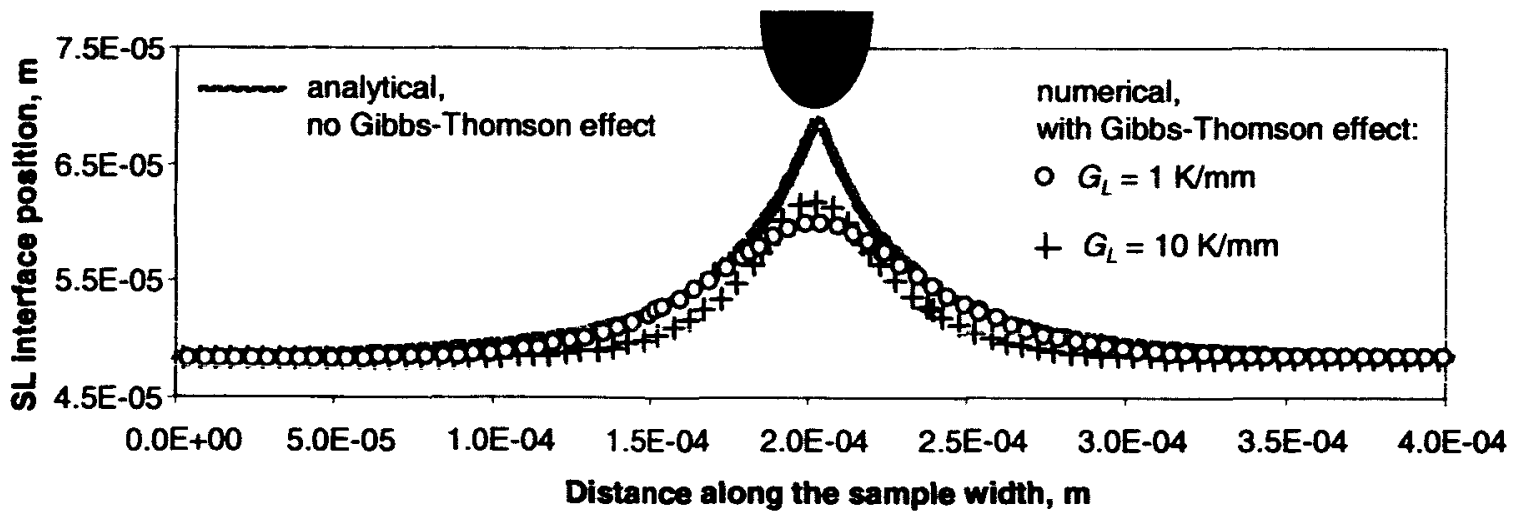

Fig. 8-Influence of the Gibbs-Thomson effect and the temperature gradient, $\mathrm{G}_{\mathrm{L}}$, on the shape of the SL interface (pure $\mathrm{Al}, \mathrm{ZrO} \mathrm{O}_{2}$ particle, $R_{P}=22.5 \mu \mathrm{m}$, $V=2 \mu \mathrm{m} / \mathrm{s}$ ). The particle shape appears noncircular because nonequal scales were used on the vertical and horizontal directions to amplify the interface deformation.

solution to this problem ${ }^{[35]}$ shows that the concentration profile, $C_{S}(z)$, in the solidified sample can be expressed as

$$
\begin{aligned}
& C_{S}(z)=\frac{C_{0}}{2}\left\{1+\operatorname{erf}\left[\frac{\sqrt{\left(V / D_{L}\right) \cdot z}}{2}\right]\right. \\
& \left.\quad+(2 k-1) \cdot e^{k(k-1)\left(V / D_{L}\right) \cdot z} \cdot \operatorname{erfc}\left[\frac{2 k-1}{2} \sqrt{\left(V / D_{L}\right) \cdot z}\right]\right\}
\end{aligned}
$$

where $C_{0}$ is the initial concentration in the liquid sample, $z$ is the distance measured from the beginning of the sample along the solidification direction, and erf and erfc are the error and complementary error functions, respectively.

The results shown in Figure 9 are for $\mathrm{Sn}-0.5$ at. pet $\mathrm{Bi}$ alloy at $V=4.9 \mu \mathrm{m} / \mathrm{s}$ and a thermal gradient in the liquid $G_{L}=17.3 \mathrm{~K} / \mathrm{mm}$. The numerical calculations were performed for a sample of length $L=10 \mathrm{~mm}$ and width $W=20 \mu \mathrm{m}$ using a grid size of $4 \mu \mathrm{m}$. In order to maintain the imposed solidification velocity and temperature gradients for the entire duration of the calculations, the following boundary conditions for the temperature at the two ends of the sample have been used:

At the liquid end,

$$
\frac{\partial T}{\partial t}=-\left(V \cdot G_{L}+m_{L} \frac{\partial C_{L}^{I}}{\partial t}\right)
$$

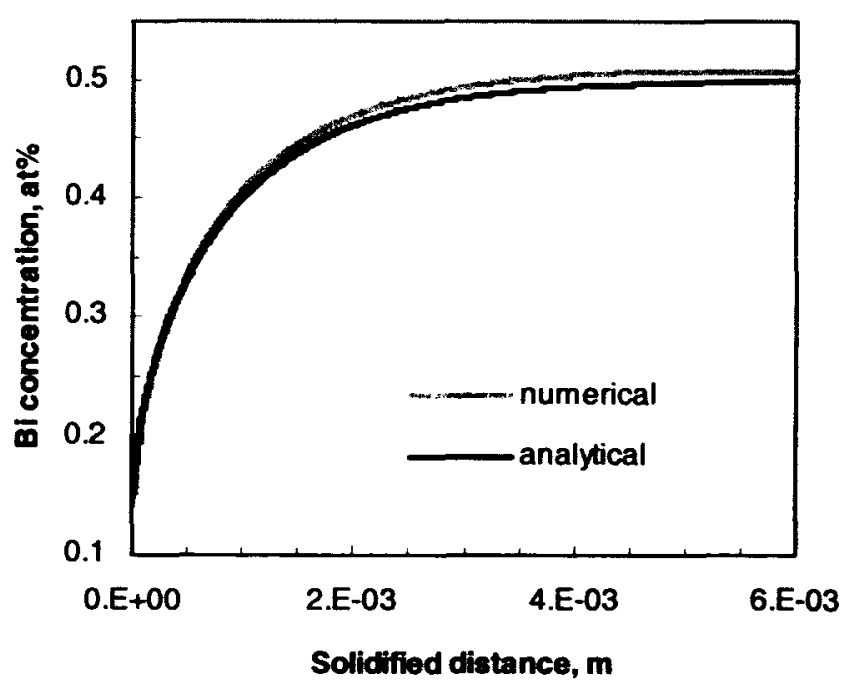

Fig. 9-Numerical predictions vs analytical solution ${ }^{[35]}$ for Bi segregation during the initial transient plane front solidification $(V=4.9 \mu \mathrm{m} / \mathrm{s})$ of $\mathrm{Sn}-0.5$ at. pet $\mathrm{Bi}$.

At the solid end,

$$
\frac{\partial T}{\partial t}=-\left(V \cdot G_{S}+m_{L} \frac{\partial C_{L}^{I}}{\partial t}\right)
$$


where $\partial C_{L}^{I} / \partial t$ represents the time change of the liquid concentration at the SL interface. Insulated boundary conditions for both temperature and concentration were used on the $x$ direction (Figure 6).

A good agreement between the analytical solution and the numerical results can be observed in Figure 9. In general, the numerical solution gives slightly higher values for the concentration as compared to the analytical solution. This is because of the linear approximation used to calculate the concentration gradient at the SL interface.

With our numerical model thus validated against analytical solutions and experimental results for the solidification of binary alloys, we can proceed to study the evolution of the SL interface shape when a foreign particle is added to the melt in front of the interface. We chose $\mathrm{Al}-2$ wt pct $\mathrm{Cu}$ for the alloy and $\mathrm{ZrO}_{2}$ for the particle. A steady-state distribution for the solute and linear distribution for the temperature were considered as initial conditions. The boundary conditions for temperature are presented in Figure 6. Insulated boundary conditions on the $x$ direction as well as on the surface of the particle were added for the solute. The results are shown in Figure 10 for three values of the distance, $h_{c}$, between the center of the particle and the flat SL interface. It can be observed that in the initial stage of solidification, when the interface is relatively far from the particle, the interface forms a small bump because the thermal conductivity of the particle is smaller than that of the melt. At this stage, the solute diffusion is only slightly obstructed by the presence of the particle, and therefore, the outcome is mainly determined by the thermal field. As the solidification proceeds, however, the solute diffusion becomes more and more affected by the presence of the particle. The localized solute accumulation decreases the interface temperature and the interface begins to turn concave beneath the particle. The depth of this concavity increases as the particle/interface distance decreases. Also note that for a very small advance of the flat SL interface, the amplitude of the two humps that form on both sides of the vertical centerline increases dramatically. This is because the lateral diffusion gradually intensifies in these regions, and therefore, the solute depletion occurring around the tip of the two humps allows them to grow ever faster.

While for the solidification of pure substances our numerical calculations as well as the analytical solution ${ }^{[36]}$ show that the solidification velocity has a negligible effect on the evolution of the SL interface near a foreign particle, this conclusion cannot be extended to the solidification of alloys. The influence of the solidification velocity is shown in Figure 11. The solute buildup in the particle/interface gap becomes more significant with the increase of the solidification velocity, and therefore, the depth of the concavity formed on the SL interface beneath the particle will be higher at

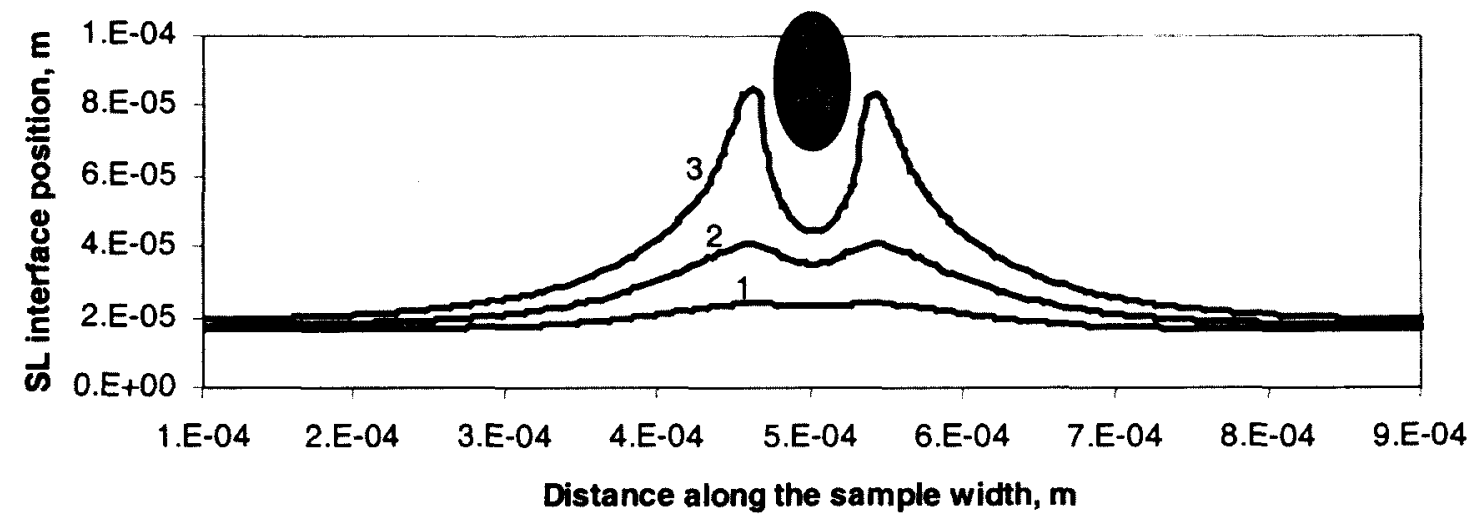

Fig. 10-Time evolution of an alloy SL interface approaching a foreign particle (Al-2 wt pct $\mathrm{Cu}, \mathrm{ZrO}_{2}$ particle, $R_{P}=22.5 \mu \mathrm{m}, V=0.2 \mu \mathrm{m} / \mathrm{s}, \mathrm{G}_{\mathrm{L}}=7 \mathrm{~K} / \mathrm{mm}$ ). For a better view of the interface deformation the particle shape appears noncircular because of nonequal scales used on the vertical and horizontal directions.

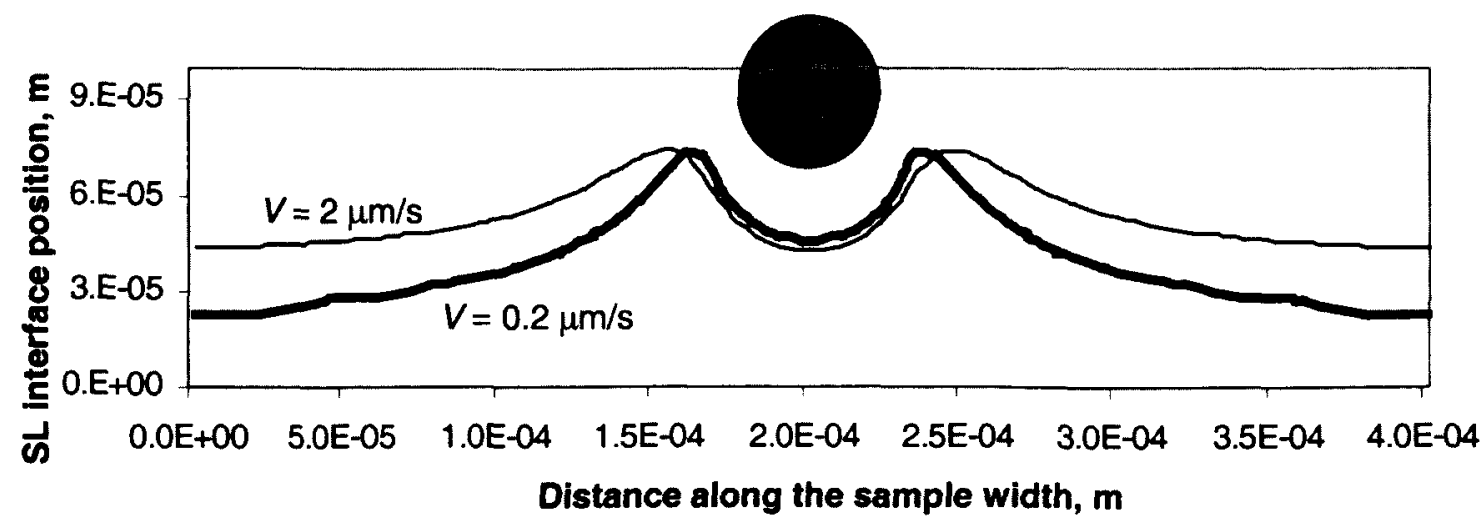

Fig. 11 -Influence of the solidification velocity on the shape of the SL interface when approaching a foreign particle $\left(\mathrm{Al}-2 \mathrm{wt}\right.$ pct $\mathrm{Cu}, \mathrm{ZrO} \mathrm{P}_{2} \mathrm{particle}, R_{P}=$ $\left.22.5 \mu \mathrm{m}, \mathrm{G}_{\mathrm{L}}=7 \mathrm{~K} / \mathrm{mm}\right)$. 
higher solidification velocity. On the other hand, when measured with respect to the flat region of the interface, the amplitude of the two humps that form on both sides of the vertical centerline is smaller at higher velocities. Thus. the solute effect becomes predominant over the thermal effect (quantified through the ratio $k_{P} / k_{L}$ ) when increasing the solidification velocity. Note that for the velocity $V=2 \mu \mathrm{m} / \mathrm{s}$, used to generate Figure 11 , the stability theory ${ }^{[37]}$ predicts that the SL interface is inherently unstable and perturbations other than those induced by the presence of the particle should also appear on the interface. This is true and we have demonstrated $^{[38]}$ that the present model has the capability to describe the development of such perturbations. For the case presented in Figure 11, however, where the sample width, $W$, was considered as only $0.4 \mathrm{~mm}$, the perturbations induced by the presence of the particle override the perturbations that would have been induced by the constitutional undercooling effect alone.

So far, our numerical model was validated against simple problems for which analytical solutions are available. It was also shown that for more complicated problems, the model provides meaningful results. However, a complete validation can be performed only against accurate experimental data. In Section IV, we will present such an experiment and compare the numerical predictions with the experimental measurements.

\section{EXPERIMENTAL APPROACH}

The principle of the experimental setup is presented in Figure 12. Projection radiography using a microfocus X-ray source offers magnification, adequate resolution, and. with suitable detector technology, adequate contrast. A state-ofthe-art submicron source with acceleration voltage of 10 to $100 \mathrm{kV}$ allows imaging of solidification of metal alloys. Real-time viewing is possible by employing an X-ray image

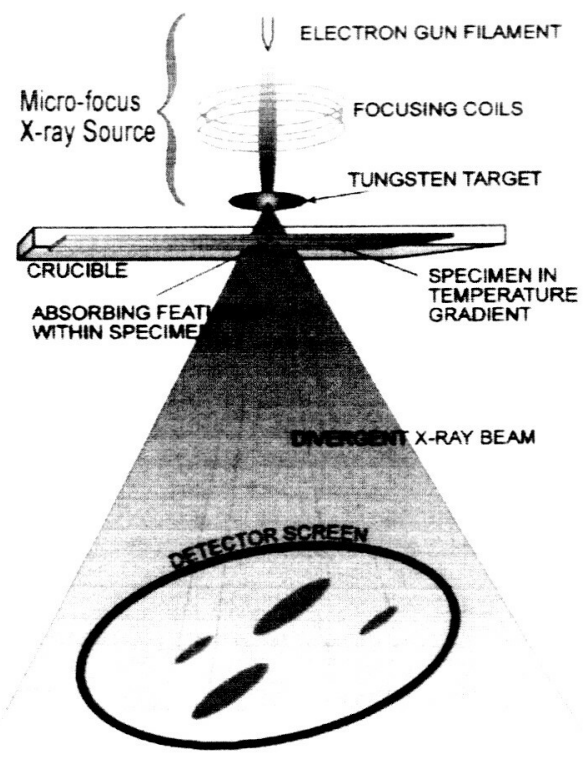

Fig. 12-A schematic illustration of the XTM apparatus and principle. ${ }^{|40|}$ intensifier and a cooled CCD camera. Resolution, which is limited by the X-ray spot size, can approach micrometer values. Radiography by projection permits placing the specimen in a furnace between the X-ray camera and the source.

The XTM furnace employed in this research is a modified horizontal Bridgman-Stockbarger design operated in air or inert gas. Samples with typical dimensions of $19 \times 4 \times$ $1 \mathrm{~mm}$ are placed in the cavity of an X-ray transparent boron nitride crucible. The sample is then covered with a boron nitride lid and placed in the furnace. A stepper motor driven screw translator translates the specimen in its crucible at rates of 0.1 to $100 \mu \mathrm{m} / \mathrm{s}$. During solidification, using the XTM, it is possible to track the interface position as a function of time and thereby obtain true interface velocity, as opposed to furnace velocity.

For this investigation, the selected sample materials were pure $\mathrm{Al}$ and $\mathrm{Al}-0.25 \mathrm{wt}$ pct $\mathrm{Au}$. The Al-Au alloy was selected because the significant density difference between the solute and the solvent provides good X-ray contrast. The samples were melted in an induction furnace and cast into cylindrical rods. $\mathrm{LECO}^{*}$ combustion measurements of the as-cast samples

LECO is a trademark of LECO Corporation. St. Joseph. MI

showed a $\mathrm{H}_{2}$ content of $0.29 \mathrm{ppm}$ in the pure $\mathrm{Al}$ sample and $0.31 \mathrm{ppm}$ in the Al-Au sample. The cylindrical castings were then cold rolled into $1-\mathrm{mm}$-thick strips and trimmed to fit into the cavity of the boron nitride crucible. The HDS experiments were performed to investigate the dynamics of the interaction between a pore and the SL interface. The processed samples were sectioned and the solute segregation pattern in the vicinity of a pore was quantified using microprobe analysis.

\section{A. Pure Aluminum}

The in-situ radiographic observation of pore growth in liquid $\mathrm{Al}$ is shown in Figure 13. The translation velocity was set at $4 \mu \mathrm{m} / \mathrm{s}$ and the thermal gradient ahead of the interface was $4.7 \mathrm{~K} / \mathrm{mm}$. Prior to its interaction with the SL interface, the pore was observed to grow with a circular cross section (Figure 13(a)). When the SL interface makes contact with the pore, the pore grows as an ellipsoid of revolution with its major axis aligned with the solidification direction

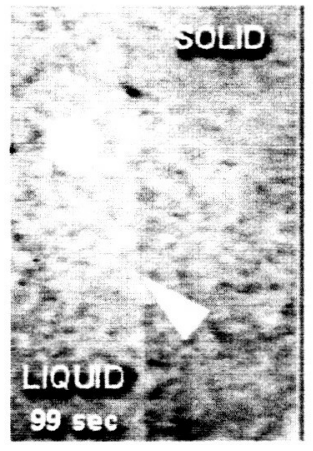

(a)

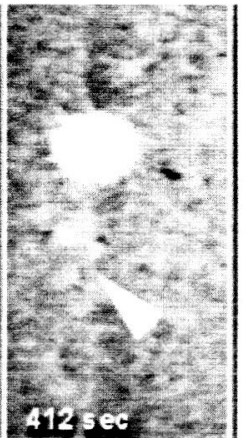

(b)

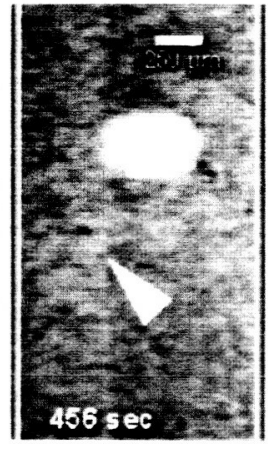

(c)
Fig. 13-Real-time radiographs of a pore and of the SL interface moving leftward: $(a)$ prior to interaction with the SL interface: and $(b)$ and $(c)$ subsequent ellipsoidal evolution during interaction with the SL interface in pure $\mathrm{Al}$. The white arrow points to the position of the SL interface. 
(Figures 13(b) and (c)). Figure 14 shows the measured time change of the volume and aspect ratio of a pore. The dashed vertical line indicates the time at which the interaction between the pore and the SL interface is initiated. It is evident from Figure 14 that the growth rate of the pore after it starts interacting with the interface is significantly higher compared to the growth rate prior to this interaction. Similar growth behavior of gas pores has also been observed in transparent organic alloys. ${ }^{[5]}$ The sudden increase of the growth rate of the pore implies that more hydrogen is available for its growth than that available through bulk diffusion in the melt. The pore acts as a sink for the $\mathrm{H}_{2}$ rejected at the solidification front.

\section{B. Al-0.25 wt pct Au Alloy}

Figure 15 shows a postsolidification radiograph of a pore after engulfment by a planar SL interface. The pore has an ellipsoidal shape with a size of about $800 \mu \mathrm{m}$ along the major axis and $660 \mu \mathrm{m}$ along the minor axis. The growth velocity was $5 \mu \mathrm{m} / \mathrm{s}$ and the temperature gradient $4.7 \mathrm{~K} / \mathrm{mm}$.

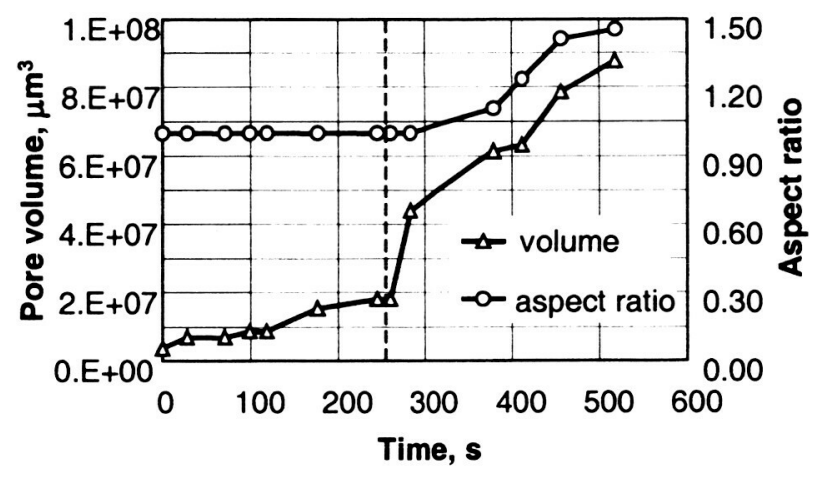

Fig. 14 Experimentally measured change in pore volume and aspect ratio as a function of time in pure $\mathrm{Al}$.
The purpose of this experiment was to investigate the effect of the pore on the solutal segregation pattern. The radiograph shows that there is a strong segregation of $\mathrm{Au}$ around the pore just prior to engulfment. The scanning electron micrograph (Figure 15(b)) shows that the microstructure in this area consists of eutectic colonies. The eutectic structure inside one such colony is shown in Figure 15(c). The thickness of this eutectic "band" varies between 70 and $80 \mu \mathrm{m}$. The centerline microprobe scan indicates that the Au composition in this region is approximately $8 \mathrm{wt}$ pct. This is in agreement with the equilibrium phase diagram for the $\mathrm{Al}-\mathrm{Au}$ system, which shows a eutectic transformation at $7.46 \mathrm{wt}$ pct $\mathrm{Au}$. Subsequent to engulfment of the pore, a comet tailshaped segregation pattern is clearly evident on the left of the pore (Figure 15(a)). The mechanism of formation of this strongly segregated region will be explained in Section V.

\section{NUMERICAL CALCULATIONS VS EXPERIMENTAL MEASUREMENTS}

At the outset, it should be mentioned that at the present stage, the model does not have the capability to account for changes of pore size and shape during the interaction with the SL interface. Therefore, the pores have been assumed to be spherical and of constant radius.

Figure 16 shows the calculated shape of the SL interface in various stages of $\mathrm{a} \mathrm{H}_{2}$ pore engulfment by the solid phase. The occurrence of a trough on the SL interface after engulfment is observed. This is typical, as suggested by Eq. [Id], for the situations when the thermal conductivity of the particle $\left(\mathrm{H}_{2}\right.$ pore in this particular case $)$ is smaller than that of the metallic matrix. As can be seen in Figure 16, once the pore is completely engulfed, the two segments of the interface are again joined together. The model handles this special situation as follows: on either side of the pore, the coordinates of the intersection of the SL interface and the pore are tracked during the engulfment of the pore. In
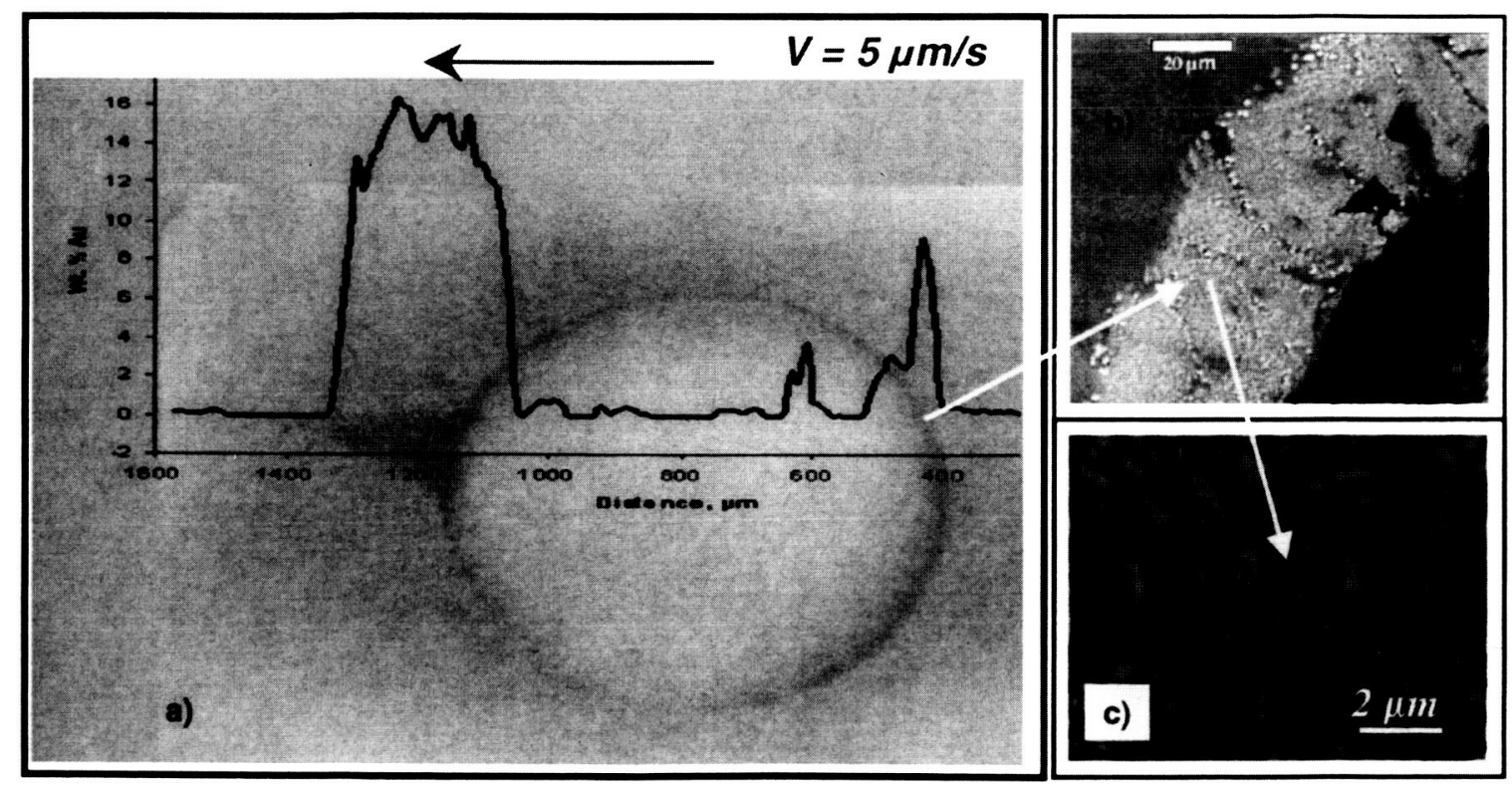

Fig. 15- (a) through (c) Postsolidification radiograph showing segregation of Au around the porosity. Superimposed is a centerline microprobe scan. 


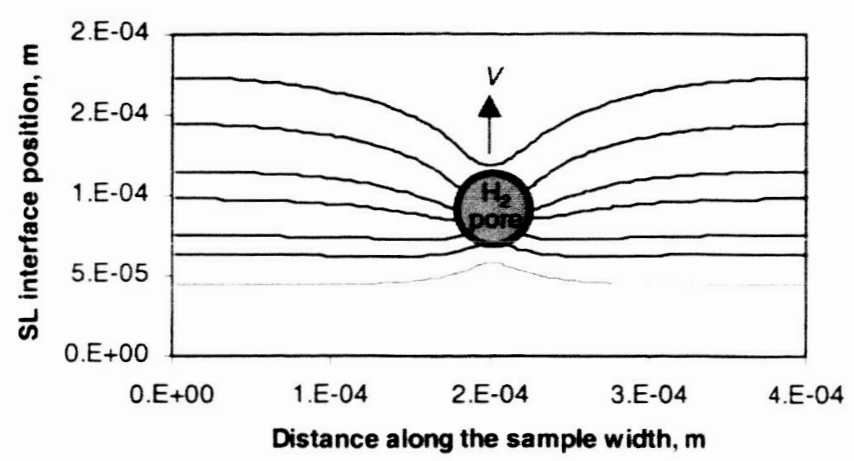

Fig. 16-Numerically calculated shape of the SL interface in various stages of engulfing a $\mathrm{H}_{2}$ pore of fixed size (pure Al. $R_{\text {pore }}=22.5 \mu \mathrm{m} . V=4 \mu \mathrm{m} / \mathrm{s}$, $\mathrm{G}_{\mathrm{L}}=4.7 \mathrm{~K} / \mathrm{mm}$ ).

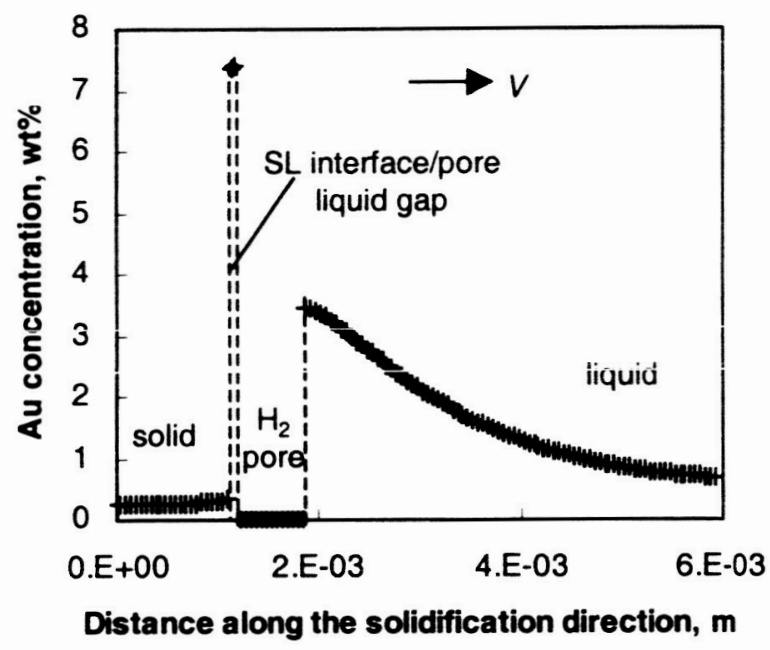

Fig. 17-Numerically calculated Au distribution profile along the sample centerline showing solute trapping in the SL interface/pore gap (Al-0.25 wt pct Au, $V=5 \mu \mathrm{m} / \mathrm{s}, \mathrm{G}_{\mathrm{L}}=4.7 \mathrm{~K} / \mathrm{mm}$. $R_{\text {pore }}=330 \mu \mathrm{m}$ ).

this model, the adopted criterion for the complete engulfment is the crossover of these two points. When the crossover occurs, a new interface marker is generated at the point of intersection.

For the solidification of the Al-0.25 wt pct Au alloy, our calculations also predict that at a distance from the pore similar to that observed experimentally, the Au concentration raises to the eutectic value, as shown in Figure 17. At the time of this publication, a model for eutectic solidification was not yet implemented in the numerical program, and therefore, the computation was stopped when the Au concentration in the liquid pore/SL interface gap reached the eutectic value. This precluded a quantitative prediction of the second region of strong Au segregation developed in front of the pore after its complete engulfment. Nevertheless, based on the interface shape of pure Al (Figure 16), it is reasonable to assume that prior to the complete engulfment, a dramatic increase of solute concentration occurs in front of the pore. This is because the solute diffusion is now directed toward the centerline of the sample, as shown in Figure 18(a). Therefore, the solidification in this region is delayed. Consequently, the shape of the SL interface at the moment of complete engulfment will look as shown in Fig-
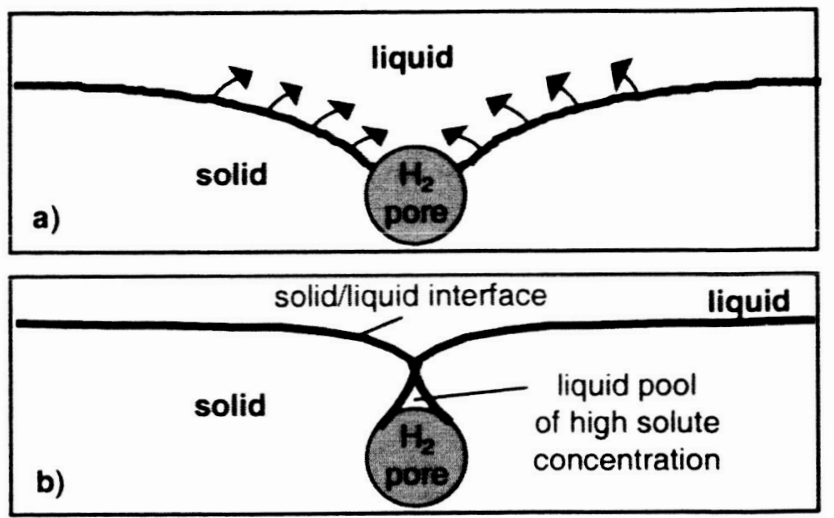

Fig. 18-The mechanism of the comet tail-shaped segregation region in front of the $\mathrm{H}_{2}$ pore: (a) solute diffusion toward the sample centerline before complete engulfment: and $(b)$ solute trapping in front of the pore at the time of complete engulfment.

ure 18(b), thus explaining the comet tail-shaped region of high $\mathrm{Au}$ concentration, which appears in Figure 15.

\section{SUMMARY}

Real-time experimental observations of the interaction between a $\mathrm{H}_{2}$ pore and a planar SL interface as well as a numerical mathematical model that explains these observations have been presented in this article.

The real-time measurements of pore growth in pure $\mathrm{Al}$ have revealed the existence of two growth regimes, as follows.

1. When the pore is relatively far from the SL interface, the mechanism of pore growth is the hydrogen diffusion through the liquid phase. The growth rate increases as the SL interface approaches the pore. At this stage, the pore has a circular cross section.

2. A sudden increase of the pore growth rate occurs when the solutal (i.e., hydrogen) field ahead of the SL interface begins interacting with the pore. The pore begins to assume an ellipsoidal shape as the SL interface gradually engulfs it.

For the solidification of an $\mathrm{Al}-0.25$ wt pct Au alloy, both the X-ray imaging and the microprobe analysis have demonstrated the development of two distinct regions of high solute concentration around the pore. The two regions have different shapes because the mechanism of their formation is also different. Chronologically, the first region develops in front of the pore as a result of solute built up in the pore/SL interface gap. It follows the contour of the pore and, therefore, develops a moonlike shape. The second region develops behind the pore, where, initially, the solidification is delayed mainly because of the thermal conductivity effect (i.e., the thermal conductivity of the pore is smaller than that of the solid phase growing around it). The solute diffusing toward this region further accentuates the solidification delay. Thus, the SL interface is forced to detach from the pore while it continues to advance at a certain angle toward the major pore axis where bridging eventually occurs. The liquid pool left behind the location where the SL interface bridging takes place is rich in solute and has a conical shape. 
Consequently, porosities in castings act not only as material discontinuities that negatively affect the mechanical properties, but also contribute to the development of regions of strong solute segregation, which further accentuates the detrimental effect on the casting properties.

A numerical mathematical model aiming to explain the experimental observations has been developed. It has the capability to track sharp and arbitrarily shaped SL interfaces during solidification of pure substances and binary alloys. Any local interface temperature change because of Gibbs-Thomson effect or solute redistribution between the solid and the liquid phase can be accommodated. For a number of relatively simple problems, the numerical predictions proved to be in good agreement with the available analytical solutions.

With regard to the interaction between the SL interface and a nonmetallic inclusion (gas pore or ceramic particle), the model can accurately describe the change of the interface shape during this interaction. It was shown that the local SL interface dynamics in the vicinity of a nonmetallic inclusion are influenced not only by the thermal conductivity ratio, $k_{P} / k_{L}$, but also by the imposed temperature gradient and solidification velocity. The solute segregation pattern developing around such an inclusion can also be described by the model.

The numerical calculations were performed on a LINUX platform with AMD Athlon $2000 \mathrm{MP}$ processor, $1.67 \mathrm{GHz}$. For a typical grid size of $\Delta y=3.5 \mu \mathrm{m}, \mathrm{V}=2 \mu \mathrm{m} / \mathrm{s}$, and $\alpha_{L}=$ $37 \cdot 10^{-6} \mathrm{~m}^{2} / \mathrm{s}$, the average computation time, required to simulate 1 seconds of real time, is approximately $\left(1.8 \cdot N \cdot 10^{-3}+6\right)$ seconds, where $N$ is the total number of the grid points.

\section{ACKNOWLEDGMENTS}

This work was supported by NASA's Biological and Physical Research Division through Grant No. NAS8-39715.

\section{APPENDIX I}

\section{Analytical solution for the shape of the solid/liquid interface in the vicinity of a particle}

Consider a liquid phase of thermal conductivity $k_{L}$ in which the thermal field is characterized by constant temperature gradient, $G_{L}$. If a foreign particle of radius $R_{P}$ and thermal conductivity $k_{P}$ is added to this liquid, then, for a two-dimensional case, the thermal field around this particle can be described by the relationship ${ }^{[36]}$

$$
T(x, z)=T_{M}+G_{L}\left[z-k_{a} \cdot R_{P}^{2} \frac{h_{c}-z}{x^{2}+\left(h_{c}-z\right)^{2}}\right]
$$

where $T$ is temperature, $x$ and $z$ are the cartesian coordinates, $T_{M}$ is the melting point, and $k_{a}$ is given by

$$
k_{a}=\frac{k_{L}-k_{P}}{k_{L}+k_{P}}
$$

Also, in Eq. [Ia], it was considered that the center of the particle is located at the position $x=0$ and $z=h_{c}$ (Eq. [Ia]). Therefore, in the absence of the particle (or if $k_{P}=k_{L}$ ), the temperature in the plane situated at $z=0$ is equal to $T_{M}$, that is, the SL interface coincides with this plane.
For the solidification of a pure substance, the local temperature, $T_{I}(x)$, of the SL interface is given by the relationship

$$
T_{I}(x)=T_{M}+\Gamma \cdot K(x)
$$

where $\Gamma$ is the Gibbs-Thomson coefficient and $K(x)$ is the local curvature of the interface. From Eqs. [Ia] and [Ic], it follows that the shape, $Z_{I}(x)$, of the SL interface can be calculated with the equation

$$
Z_{I}(x)-k_{a} \cdot R_{P}^{2} \frac{h_{c}-Z_{I}(x)}{x^{2}+\left[h_{c}-Z_{I}(x)\right]^{2}}=\frac{\Gamma \cdot K(x)}{G_{L}}
$$

Equation [Id] predicts that if $k_{P} \neq k_{L}$, the SL interface will be planar only at $x \rightarrow \infty$, while in the vicinity of the particle, it will be concave or convex, depending on the ratio $k_{P} / k_{L}$. If $k_{P} / k_{L}<1$ (i.e., $k_{a}>0$ ), the interface will be convex toward the particle, as shown in Eq. [Ia], and concave if $k_{P} / k_{L}>1$ (i.e., $k_{a}<0$ ). It turns out that, because of the presence of the curvature term, $K(x)$, which is given by Eq. [8], the general solution of Eq. [Id] is difficult to find. However, for a sufficiently high temperature gradient, $G_{L}$, the right-hand side of Eq. [Id] becomes negligible and an approximate solution can easily be obtained. Obviously, this solution is exact only for the imaginary case when $\Gamma=0$ (i.e., no Gibbs-Thomson effect).

Inspection of Eq. [Id] suggests that by choosing the reference length scale

$$
L_{\mathrm{ref}}=\sqrt{\left|k_{a}\right|} \cdot R_{P}
$$

where $\left|k_{a}\right|$ is the absolute value of the parameter $k_{a}$, Eq. [Id] can be written in nondimensional form as

$\hat{K}(\hat{x})=\frac{G_{L}\left|k_{a}\right| R_{P}}{\Gamma} \cdot\left[\hat{Z}_{I}(\hat{x})-\operatorname{sgn}\left(k_{a}\right) \cdot \frac{\hat{h}_{c}-\hat{Z}_{I}(\hat{x})}{(\hat{x})^{2}+\left(\hat{h}_{c}-\hat{Z}_{I}(\hat{x})\right)^{2}}\right]$

The sign "^" in Eq. [If] indicates nondimensional quantities, while $\operatorname{sgn}\left(k_{a}\right)$ means the algebraic sign of $k_{a}$.

Equation [If] shows that the influence of the material and process parameters (i.e., $G_{L}, k_{a}, \Gamma$, and $R_{P}$ ) on the local SL interface curvature can be completely separated from the local interface coordinates when choosing the reference lengthscale, as given by Eq. [Ie]. Thus, it seems that the natural lengthscale for this type of problems is not particle radius, $R_{P}$, but $\sqrt{\left|k_{a}\right|} R_{P}$. This reference lengthscale has been adopted in this work.

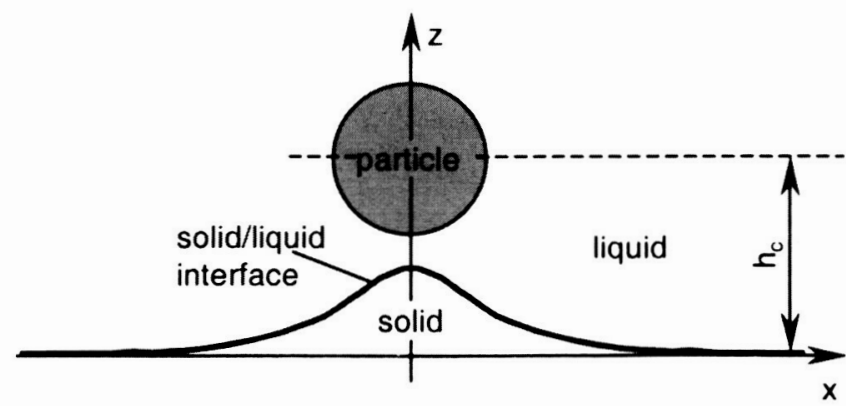

Fig. Ia-Nonplanar SL interface in the presence of a foreign particle $\left(k_{p}<k_{L}\right)$. 


\section{APPENDIX II}

The effect of the grid size on the calculated SL interface curvature

One of the drawbacks of the interface tracking method presented in this article is that the accuracy to which the local SL interface curvature is calculated depends on the choice of the grid size. An illustration of the expected level of errors introduced by a certain grid size is presented in this Appendix.

In Appendix I, it was shown that at a very high-temperature gradient, $G_{L}$, or in the hypothetical case of $\Gamma=0$, an exact analytical solution can be obtained for the SL interface by means of Eq. [Id]. The corresponding exact curvature can also be calculated at any point from the same equation. It can be shown that the minimum interface curvature radius, $R_{I, \min }$, occurs when the SL interface first touches the particle at $x=0$ (Eq. [Ia]) and is given by

$$
R_{I, \min }=\left|\frac{k_{a}-1}{k_{a}}\right| \frac{R_{P}}{2}
$$

For the particular case of an $\mathrm{H}_{2}$ pore in a liquid $\mathrm{Al}$ matrix $\left(k_{a}=0.991\right)$ and $R_{P}=22.5 \mu \mathrm{m}$, Eq. [IIa] gives a curvature radius $R_{I}=0.102 \mu \mathrm{m}$. This is a very disadvantageous case, because in order to minimize the errors introduced by the numerical calculation of the curvature, a grid size smaller than $0.1 \mu \mathrm{m}$ would be necessary. In reality, however, the Gibbs-Thomson effect is always present and the result is that $R_{I, \text { min }}$ is larger than that predicted by Eq. [IIa]. A recently developed procedure ${ }^{[39]}$ shows that for this particular case, a more realistic value is $R_{l, \text { min }}=8 \mu \mathrm{m}$, which closely corresponds to the hypothetical case of $\Gamma=0, k_{P}=$ $24.92 \mathrm{~W} / \mathrm{m} \cdot \mathrm{K}$, and keeping unchanged all the other parameters. Thus, even for this very disadvantageous case (i.e., $k_{P} \ll k_{L}$ ), the minimum interface curvature radius is no less than $R_{p} / 3$.

Further, in order to test the influence of the gird size on the curvature calculated by means of the cubic spline method, the following procedure has been used in this work.

1. At each location, $x=\left(X_{i}+X_{i+1}\right) / 2$, where $X_{i}$ is the position of the $i$ th grid line; the interface position, $Z_{I(x)}$, is calculated from Eq. [Id], for $\Gamma=0$ and the newly calculated $k_{P}$. Note that the grid size on the $x$ direction is given by the difference $\left(X_{i+1}-X_{i}\right)$, and the interface marker points thus calculated do not coincide with the grid lines.

2. Use the cubic spline interpolation procedure to position the interface marker points on the grid lines and calculate the curvature, $K_{i}$, at each such point.

3. Calculated the curvature error, $\mathrm{Err}_{i}$, as

$$
\operatorname{Err}_{i}=100 \cdot\left|\frac{K_{i}-K_{i}^{*}}{K_{i}^{*}}\right|
$$

where $K_{i}^{*}$ is the exact curvature at the $i$ th marker point, calculated from Eq. [Id].

An analysis of the errors thus calculated indicated that the highest deviations occured near the inflexion points of the interface and at the point of minimum curvature radius (i.e., at $x=0$ in Eq. [Ia]). The deviations at the inflexion points can be as high as 2.5 times larger than those occurring at the point of minimum curvature radius. However, near the

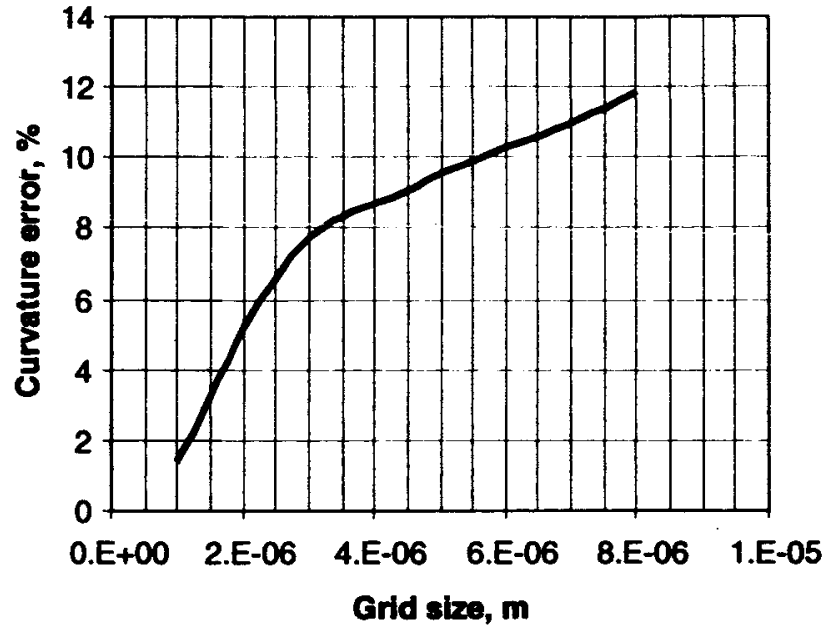

Fig. Ila-Calculated curvature error at the point of minimum interface curvature radius $\left(R_{P}=22.5 \mu \mathrm{m}, k_{L}=95 \mathrm{~W} / \mathrm{m} \cdot \mathrm{K}, k_{p}=24.92 \mathrm{~W} / \mathrm{m} \cdot \mathrm{K}, \Gamma=0 \mathrm{~K} \cdot \mathrm{m}\right)$.

inflexion points, the curvature assumes relatively low values, and therefore, the numerical errors introduced at these points do not significantly influence the interface motion. Consequently, the attention should be focused at the point of minimum curvature radius. A plot of the curvature error occurring at this point $v s$ grid size is presented in Figure [Ha]. Thus, for a grid size smaller than $R_{P} / 6$, i.e., smaller than half of the minimum curvature radius, Fig. [IIa] indicates that the errors will be smaller than 8.5 pct.

\section{LIST OF SYMBOLS}

$A_{1}, A_{2}, A_{3}, Q$ quantities defined in Eqs. [18] through [20] to calculate the normal growth velocity

$b_{E}, b_{N}, b_{S}, \quad$ coefficients in the discrete form of the $b_{x}, b_{y}, b_{w} \quad$ transport equation of field variables

$C \quad$ concentration

$C_{L}, C_{S} \quad$ liquid and solid concentrations, respectively $C_{P} \quad$ volumetric specific heat

$D_{L}, D_{S} \quad$ solute diffusion coefficient in the liquid and solid phases, respectively

$\Phi \quad$ generalized field variable

$f \quad$ coefficient that takes the value of 0.5

$G_{L}, G_{S} \quad$ temperature gradient in the liquid and solid, respectively

$\Delta H_{f} \quad$ latent heat of fusion

$h_{c} \quad$ distance between the planar interface and the center of the particle

$k \quad$ partition coefficient

$k_{L}, k_{S}, k_{P} \quad$ thermal conductivities of the liquid, solid, and particle, respectively

$\alpha_{L}, \alpha_{S} \quad$ thermal diffusivities of the liquid and solid, respectively

$K \quad$ solid/liquid interface curvature

$\Gamma \quad$ Gibbs-Thomson coefficient

$N_{1}, N_{2}, \quad$ shape functions used for the interpolation of $N_{3}, N_{4}$

$\mathbf{N}$ the field variables

unit normal vector

$\Delta n \quad$ length of the probe projected along the normal direction

$m_{L} \quad$ liquidus slope 
$V_{n}$

$x, z$

particle radius

temperature

melting temperature

temperature in the liquid and solid phases, respectively time, time-step size

imposed growth velocity through external boundary conditions

\section{$\Xi$} normal growth velocity

Subscripts/Superscripts

$L \quad$ pertaining to the liquid phase

$S \quad$ pertaining to the solid phase

$i \quad$ at the solid/liquid interface

$o \quad$ at the beginning of the time-step

$n L, n S$ on the normal direction in the liquid and solid phases, respectively

\section{REFERENCES}

1. D.M. Stefanescu: The Science and Engineering of Casting Solidification, Kluwer Academic/Plenum Publishers, New York 2002.

2. H. Fredriksson and L. Svensson: Metall. Trans. B, 1976, vol. 7B, pp. 599-06.

3. J.M. Kim, D.G. Kim, H.W. Kwon, and C.R. Loper, Jr.: Scripta Mater., 1998, vol. 39 (7), pp. 969-75.

4. P.D. Lee and J.D. Hunt: Modeling of Casting, Welding, and Advanced Solidification Processes VII, M. Cross and L. Campbell, eds., TMS, Warrendale, PA, 1995, pp. 585-92.

5. H. Jamgotchian, R. Trivedi, and B. Billa: J. Cryst. Growth, 1993 , vol. 134, pp. 181-95.

6. D. Shangguan and D.M. Stefanescu: Metall. Trans. B, 1991, vol. 22B, pp. $385-88$.

7. T.S. Piwonka and M.C. Flemings: Trans. TMS-AIME, 1966, vol. 236, pp. 1157-65.

8. D.R. Poirier, K. Yeum, and A.L. Maples: Metall. Trans. A, 1987, vol. 18A, pp. 1979-87.

9. P.D. Lee and J.D. Hunt: Acta Mater., 1997, vol. 45 (10), pp. 4155-69.

10. V.R. Voller and S. Sundarraj: Int. J. Heat Mass Transfer, 1995, vol. 38 (6), pp. 1009-18.

11. P. Rousset, M. Rappaz, and B. Hannart: Metall. Mater. Trans. A, 1995, vol. 26A, pp. 2349-58.

12. L.N. Brush and R.F. Sekerka: J. Cryst. Growth, 1989, vol. 96, pp. 419-41.

13. M. Lacroix: Num. Heat Transfer B, 1989, vol. 15, pp. 191-210.

14. C.H. Kuo and W.C. Schreiber: HTD, 1994, vol. 275, pp. 57-64.

15. S.L. Wang, R.F. Sekerka, A.A. Weeler, B.T. Murray, S.R. Coriel, R.J. Braun, and G.B. McFadden: Physica D, 1993, vol. 69, pp. 189-200.

16. J.A. Warren and W.J. Boettinger: Acta Metall. Mater., 1995, vol. 43 (2), pp. 689-703.

17. W.J. Boettinger and J.A. Warren: Metall. Mater. Trans. A, 1996, vol, 27A, pp. 657-69.

18. S. Osher and J. Sethian: J. Comp. Phys., 1988, vol. 79, pp. 12-49.

19. J.A. Sethian and J. Strain: J. Comp. Phys., 1992, vol. 98, pp. 231-53.

20. A. Karma and W.-J. Rappel: Phys. Rev. E, 1996, vol. 53 (4), pp. R3017-20.

21. A. Karma: Phys. Rev. Lett., 2001, vol. 87, 115701, pp.1-4

22. B. Merriman, J.K. Bence, and S.J. Osher: J. Comp. Phys., 1994, vol. 112, pp. 334-63.
23. M. Sussman, P. Smereka, and S. Osher: J. Comp. Phys., 1994, vol. 114, pp. 146-59.

24. T.Y. Hou, Z. Li, S. Osher, and H. Zhao: J. Comp. Phys., 1997, vol. 134 , pp. 236-52.

25. S. Chen, B. Merriman, S. Osher, and P. Smereka: J. Comp. Phys., 1997, vol. 135, pp. 8-29.

26. D. Peng, B. Merriman, S. Osher, H. Zhao, and M. Kang: J. Comp. Phys., 1999, vol. 155, pp. 410-38.

27. Yung-Tae Kim, N. Goldenfeld, and J. Dantzig: Phys. Rev. E, 2000, vol. $62(2)$, pp. 2471-74.

28. W. Shyy, H.S. Udaykumar, M.M. Rao, and R.W. Smith: Computational Fluid Dynamics with Moving Boundaries, Taylor \& Francis, Washington, DC, 1996.

29. H.S. Udaykumar and W. Shyy: Num. Heat Transfer B, 1995, vol. 27, pp. 127-53.

30. H.S. Udaykumar and W. Shyy: Int. J. Heat Mass Transfer, 1995, vol. 38 (11), pp. 2057-73.

31. A.V. Catalina: Ph.D. Dissertation, The University of Alabama, Tuscaloosa, AL, 2000.

32. A.V. Catalina and D.M. Stefanescu: Modeling of Casting and Solidification Processes 1999, C.P. Hong, J.K. Choi, and D.H. Kim, eds., Yonsei University pub., Korea, Seoul, 2000, pp. 3-11.

33. W.H. Press, S.A. Teukolsky, W.T. Vetterling, and B.P. Flannery: Numerical Recipes in C, Cambridge University Press, Cambridge, United Kingdom, 1986, p. 113.

34. R.D. Cook, D.S. Malkus, and M.E. Plesha: Concepts and Applications of Finite Element Analysis, John Wiley \& Sons, New York, NY, 1989, pp. 98-99 and 166-67.

35. V.G. Smith, W.A. Tiller, and J.W. Rutter: Can. J. Phys., 1955, vol. 33, pp. $723-45$.

36. A.V. Catalina, S. Mukherjee, and D.M. Stefanescu: Metall. Mater. Trans. A, 2000, vol. 31A, pp. 2559-68.

37. W.W. Mullins and R.F. Sekerka: J. Appl. Phys., 1964, vol. 35 (2), pp. 444-51.

38. A.V. Cataina, S. Sen, D.M. Stefanescu, and P.A. Curreri: Modeling of Casting, Welding and Advanced Solidification Processes IX, P.R. Sahm, P.N. Hansen, and J.G. Conley eds., Shaker Verlag, Aachen, Germany, 2000, pp. 445-52.

39. A.V. Catalina, D.M. Stefanescu, and S. Sen: Modeling of Casting, Welding and Advanced Solidification Processes X, D.M. Stefanescu, J. Warren, M. Jolly, and M. Krane eds.,TMS, Warrendale, PA, 2003, pp. 125-32.

40. S. Sen, W.F. Kaukler, P.A. Curreri, and B.K. Dhindaw: Proc. 6th Asian Foundry Congr., A.K. Chakrabarti, B.K. Dhindaw, G.L. Datta, and C.S. Sivaramakrishnan, eds., The Institute of Indian Foundrymen, Calcutta, 1999, pp. 303-08.

41. W. Kurz and D.J. Fisher: Fundamentals of Solidification, 2nd ed., Trans Tech Publications, Aedermannsdorf, Switzerland, 1986, pp. 240-41.

42. CRC Handbook of Chemistry and Physics, 67th ed., R.C. West, ed., CRC Press Inc., Boca Raton, FL, 1986.

43. Binary Alloy Phase Diagrams, T.B. Massalski, ed., ASM, Metals Park, OH, 1987, p. 90.

44. Thermophysical Properties of Matter, Y.S. Touloukian, ed.. The Macmillan Company, New York, NY, vol. 4.

45. J. Forsten and H.M. Miekkoja: J. Inst. Met., 1967, vol. 95, pp. 143-45.

46. Thermophysical Properties of High Temperature Solid Materials, Y.S. Touloukian, ed., The Macmillan Company, New York, NY, vol. 4.

47. D.R. Poirier and G.H. Geiger: Transport Phenomena in Materials Processing, TMS, Warrendale, PA, 1994, pp. 192 and 620.

48. Engineering Properties of Selected Ceramic Materials, J.F. Lynch, C.G. Ruderer, and W.H. Duckworth, eds., The American Ceramic Society, Inc., Columbus, OH, 1966. 